\title{
Coherent Electron Hole Dynamics Near a Conical Intersection
}

\author{
Henry Timmers, ${ }^{1}$ Zheng Li, ${ }^{2,3}$ Niranjan Shivaram, ${ }^{1}$ Robin Santra, ${ }^{2,3}$ Oriol Vendrell, ${ }^{2}$ and Arvinder Sandhu ${ }^{1, *}$ \\ ${ }^{1}$ Department of Physics, University of Arizona, Tucson, Arizona 85721, USA \\ ${ }^{2}$ Center for Free-Electron Laser Science, DESY, Notkestrasse 85, D-22607 Hamburg, Germany \\ ${ }^{3}$ Department of Physics, University of Hamburg, D-20355 Hamburg, Germany
}

(Received 24 June 2014; published 12 September 2014)

\begin{abstract}
The coherent evolution of an electron hole in a photoionized molecule represents an unexplored facet of charge transfer phenomena occurring in complex systems. Using ultrafast extreme ultraviolet spectroscopy, we investigate the real-time dynamics of an electron hole wave packet created near a conical intersection in $\mathrm{CO}_{2}$. We resolve the oscillation of the electron hole density between $\sigma$ and $\pi$ character, driven by the coupled bending and asymmetric stretch vibrations of the molecule. We also quantify the mixing between electron hole configurations and find that the wave packet coherence diminishes with time due to thermal dephasing.
\end{abstract}

The rapid motion of charge within a molecule and the resulting redistribution of energy is essential for the function of chemical and biochemical reactions [1,2]. Fundamentally, molecular charge dynamics are driven by either electron correlation effects or through the coupling of electronic and nuclear degrees of freedom. Therefore, the natural time scale for charge motion lies in the attosecond to femtosecond regime. Recent developments in attosecond, extreme ultraviolet (XUV) science provide new opportunities for the real-time investigation of electron dynamics in atomic [3-6] and molecular systems [7-13]. Specifically, by virtue of the high photon energy, ultrafast XUV pulses allow access to electron hole dynamics in photoionized molecules, an unexplored class of charge transfer phenomena [14-16]. Compared to the electron dynamics in neutral molecules, a photoionized molecule is an open system where additional interactions, including photoelectron entanglement [17], can influence the charge dynamics.

Ultrafast electron hole dynamics in a photoionized molecule originate from the coherent evolution of a superposition of quantum states composing a nonstationary wave packet. Conventional, synchrotron-based XUV sources can be used to infer electron hole dynamics [18,19], but this energy resolved approach cannot observe wave packet motion in real time. On the other hand, time-domain studies can elucidate the dynamic nature of correlations driving ultrafast charge dynamics in molecules and can open the door for the direct control of reaction pathways. Attosecond and femtosecond XUV pulses based on nonlinear high-harmonic generation (HHG) offer sufficiently broad bandwidths, forming an ideal tool for probing electronic superpositions in a wide variety of systems $[5,6,11,12]$. However, these techniques have not been applied in the pump-probe studies of coherent charge dynamics in polyatomic systems that exhibit complex behavior near conical intersections.

A conical intersection arises when distinct electronic states become degenerate at a certain set of interatomic coordinates [20], leading to the breakdown of the conventional Born-Oppenheimer approximation that serves as the basis for the interpretation of many molecular phenomena. Near this point of degeneracy, the electronic and vibrational degrees of freedom become strongly coupled, producing nonadiabatic, vibronic effects which serve to mediate charge transfer processes.

Owing to its small size and known structure [21-23], the $\mathrm{CO}_{2}$ molecule forms an excellent choice for studying such complex charge dynamics. Using a femtosecond XUV pump and near-infrared (NIR) probe scheme, we investigate the electron hole dynamics near a conical intersection

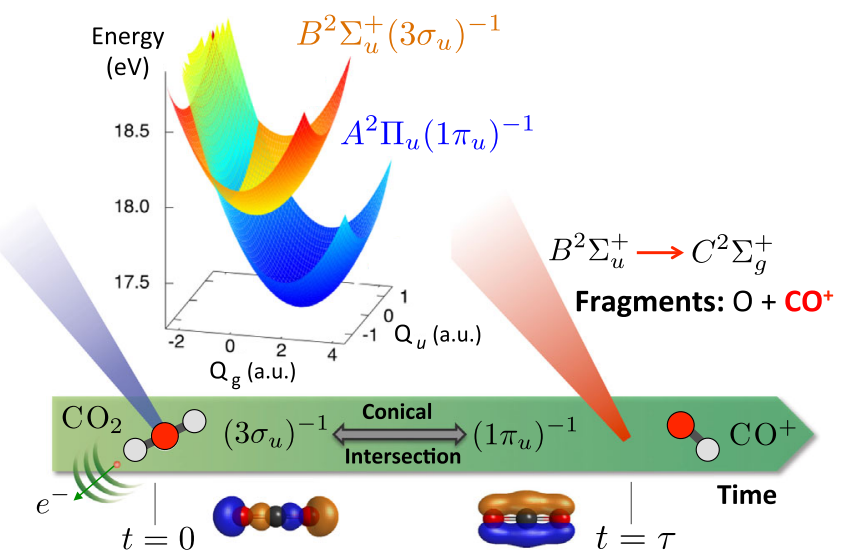

FIG. 1 (color online). The femtosecond XUV pulse photoionizes $\mathrm{CO}_{2}$ to the $B^{2} \Sigma_{u}^{+}$ionic state with $\sigma_{u}$ electron hole density distributed along the bonding axis. Vibronic interactions near the conical intersection couple this state to the $A^{2} \Pi_{u}$ ionic state which is associated with the $\pi_{u}$ electron hole. The potential energy surfaces of both these states are shown as a function of the symmetric C-O stretch, $Q_{g}$, and asymmetric C-O stretch, $Q_{u}$, coordinates. We resolve the electron hole dynamics with a time-delayed NIR pulse that excites the $B^{2} \Sigma_{u}^{+}$population to the predissociative $C^{2} \Sigma_{g}^{+}$state resulting in the formation of observable $\mathrm{CO}^{+}$ions. 
in the $\mathrm{CO}_{2}^{+}$ion. We find that the electron hole density exhibits a coherent oscillation between the $\sigma$ and $\pi$ characters. By analyzing the amplitude of this quantum beat signal, we are able to quantify the strength of the vibronic coupling between two electronic states forming a conical intersection. Importantly, these dynamics evolve in bound (nondissociative) states of the molecule, which allows us to monitor the coherence of the electron hole over a long time scale. We observe that the coherence in the electron hole oscillation decreases with time and we attribute this decay to a thermal dephasing mechanism. In essence, we obtain a comprehensive view of the complex evolution of an electron hole wave packet generated in a polyatomic molecule. Our results demonstrate that timeresolved XUV spectroscopy forms a versatile tool for the exploration of correlated dynamics of inner-valence electron holes. This represents an interesting and complex regime distinct from the prior studies of valence electron dynamics in neutral molecules [24-27]. Furthermore, our ability to quantify the nonadiabatic couplings and quantum coherence opens new avenues for the ultrafast studies of charge and energy transfer mechanisms.

The ionization from the outer four molecular orbitals of $\mathrm{CO}_{2}$ results in the formation of the ionic states $X^{2} \Pi_{g}\left(1 \pi_{g}\right)^{-1}$ (13.8 eV), $A^{2} \Pi_{u}\left(1 \pi_{u}\right)^{-1}(17.3 \mathrm{eV}), B^{2} \Sigma_{u}^{+}\left(3 \sigma_{u}\right)^{-1}(18.1 \mathrm{eV})$, and $C^{2} \Sigma_{g}^{+}\left(4 \sigma_{g}\right)^{-1}(19.4 \mathrm{eV})$ [21]. Importantly, the $A^{2} \Pi_{u}\left(1 \pi_{u}\right)^{-1}$ and $B^{2} \Sigma_{u}^{+}\left(3 \sigma_{u}\right)^{-1}$ states form a conical intersection for nuclear coordinates close to the neutral ground-state, equilibrium geometry. These states couple through the joint effect of the bending and the asymmetric stretching motions of the $\mathrm{C}-\mathrm{O}$ bonds, which is the wellknown case of bilinear vibronic coupling [22]. The potential energy surfaces of the $B^{2} \Sigma_{u}^{+}\left(3 \sigma_{u}\right)^{-1}$ and $A^{2} \Pi_{u}\left(1 \pi_{u}\right)^{-1}$ states are shown in Fig. 1 in the vicinity of the conical intersection. To study the nonadiabatic electron hole dynamics associated with these coupled states, we use the pump-probe scheme illustrated in Fig. 1. A few-femtosecond XUV pulse obtained through $\mathrm{HHG}$ in Xe gas is used to ionize the $\mathrm{CO}_{2}$ molecules to the $B^{2} \Sigma_{u}^{+}$ionic state, launching an electron hole wave packet with density along the bonding axis $\left(\sigma_{u}\right.$ hole). Vibronic interactions induced by the conical intersection will result in the periodic transfer of the electron hole population to the $A^{2} \Pi_{u}$ state, with electron hole density around the bonding axis $\left(\pi_{u}\right.$ hole). To resolve these dynamics, we use a time-delayed 45 fs NIR pulse with a central wavelength of $785 \mathrm{~nm}$ to excite the $B^{2} \Sigma_{u}^{+}$state population to the quasibound $C^{2} \Sigma_{g}^{+}$state, the dissociation of which creates observable $\mathrm{CO}^{+}$fragments. Using a time gated velocity map imaging detector, we measure the yield of low energy $\mathrm{CO}^{+}$ ions as a function of XUV-pump, NIR-probe time delay.

Figure 2(a) shows the experimental measurements of the $\mathrm{CO}^{+}$ion yield obtained as a function of pump-probe time delay. We recorded the ion yield for NIR polarizations both parallel and perpendicular to the XUV polarization. The most striking feature of this data is the strong (a)

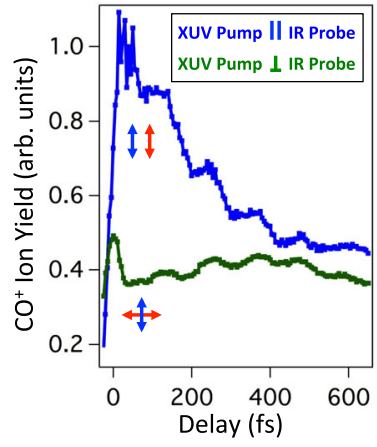

(c)
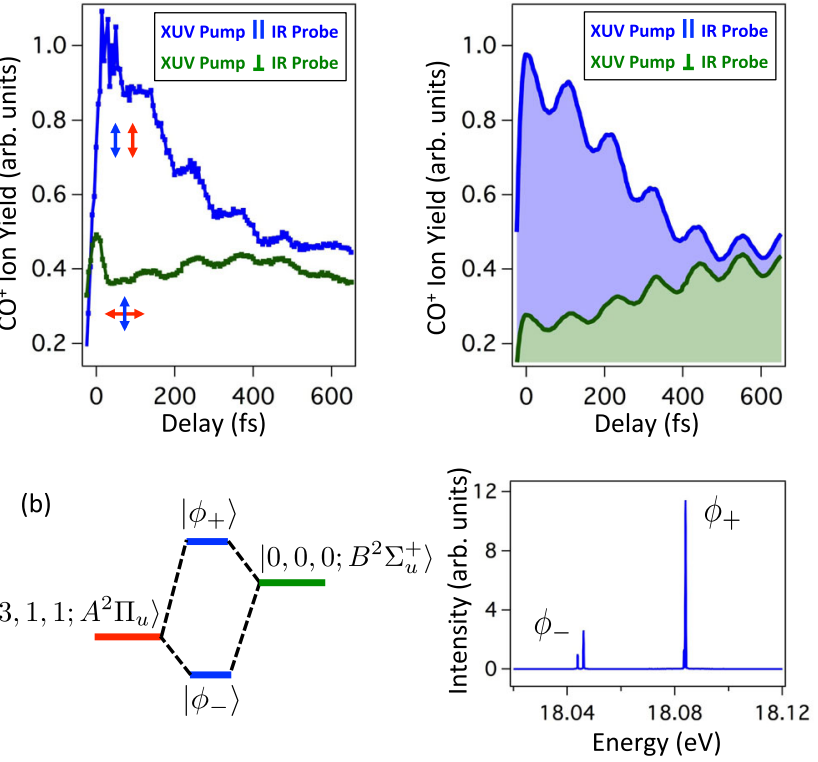

FIG. 2 (color online). (a) Experimental traces of the $\mathrm{CO}^{+}$ion yield as a function of XUV pump, NIR probe time delay for both parallel (blue) and perpendicular (green) NIR polarizations relative to the XUV field. (b) The $\phi_{+}$and $\phi_{-}$eigenstates are mostly a superposition of vibronic states $\left|0,0,0 ; B^{2} \Sigma_{u}^{+}\right\rangle$and $\left|3,1,1 ; A^{2} \Pi_{u}\right\rangle$, respectively [29]. The calculated eigenstate spectrum of the ion accessible by the XUV excitation is shown on the right. The small peak close to $\phi_{-}$is assigned to $\left|2,3,1 ; A^{2} \Pi_{u}\right\rangle$ and does not contribute to the observed signal. (c) Theoretical $\mathrm{CO}^{+}$ion traces for both parallel and perpendicular probing fields corresponding to Eq. (3). Calculations are based on a quantum treatment of the vibronic dynamics and a classical description of the rotational degrees of freedom.

oscillatory signal with a period of 115 fs riding on top of the ion yield curves. To unveil the nature of the $115 \mathrm{fs}$ oscillation, we consider the bilinear vibronic coupling Hamiltonian proposed in Ref. [22], with vibronic states $\mid n_{g}$, $\left.n_{\rho}, n_{u} ; A^{2} \Pi_{u}\right\rangle=\left|\chi_{A}\right\rangle$ and $\left|n_{g}^{\prime}, n_{\rho}^{\prime}, n_{u}^{\prime} ; B^{2} \Sigma_{u}^{+}\right\rangle=\left|\chi_{B}\right\rangle$ forming our basis set. The quantum numbers $n_{g}, n_{\rho}$, and $n_{u}$ correspond to the vibrational excitations in $\mathrm{C}-\mathrm{O}$ symmetric stretch, $\mathrm{O}-\mathrm{C}-\mathrm{O}$ bending, and $\mathrm{C}-\mathrm{O}$ asymmetric stretch modes. The motion along these three normal modes is represented using coordinates $Q_{g}, Q_{\rho}$, and $Q_{u}$, respectively. In this formalism, the coupling induced by the conical intersection manifests as off-diagonal matrix elements in the Hamiltonian, $V_{A B} \propto\left\langle\chi_{A}\left|Q_{\rho} Q_{u}\right| \chi_{B}\right\rangle$, resulting in the mixing of vibronic states. Using symmetry considerations and a multiconfigurational time-dependent Hartree (MCTDH) numerical approach [28], we find that the $\left|\chi_{B}\right\rangle=\left|0,0,0 ; B^{2} \Sigma_{u}^{+}\right\rangle$and $\left|\chi_{A}\right\rangle=\left|3,1,1 ; A^{2} \Pi_{u}\right\rangle$ vibronic states exhibit the strongest coupling and dominate the wave packet dynamics [29]. These two states are nonstationary states of the vibronic Hamiltonian. However, one can define the stationary eigenstates $\left|\phi_{ \pm}\right\rangle$by a representation transformation 


$$
\left(\begin{array}{l}
\left|\phi_{+}\right\rangle \\
\left|\phi_{-}\right\rangle
\end{array}\right)=\left(\begin{array}{cc}
\cos \theta & \sin \theta \\
-\sin \theta & \cos \theta
\end{array}\right)\left(\begin{array}{l}
\left|\chi_{B}\right\rangle \\
\left|\chi_{A}\right\rangle
\end{array}\right)
$$

where the $\theta$ is the mixing angle which quantifies the strength of coupling between the two vibronic states. The hybridization diagram corresponding to this situation is shown in Fig. 2(b).

The XUV spectrum used in our experiment predominately populates the $B^{2} \Sigma_{u}^{+}$electronic state [23,29], forming a $\sigma_{u}$ hole. This initial state is a coherent superposition of hybridized cationic eigenstates, $\left|\phi_{ \pm}\right\rangle$, whose relative populations are shown in the eigenstate spectrum of Fig. 2(b). Using the known amplitudes $a_{ \pm}$and energies $E_{ \pm}$for $\left|\phi_{ \pm}\right\rangle$ [29], we can write a time-dependent wave function for this system as

$$
|\Psi(t)\rangle=a_{+} e^{-i E_{+} t}\left|\phi_{+}\right\rangle+a_{-} e^{-i E_{-} t}\left|\phi_{-}\right\rangle .
$$

Since these eigenstates states are composed of a linear combination of $\sigma_{u}$ and $\pi_{u}$ electron hole states, the system develops a mixed $\sigma-\pi$ electronic character as the wave function evolves. We can see from the above equation that the energy separation $E_{+}-E_{-}$characterizes the time scale of quantum beating between the $\left|\phi_{+}\right\rangle$and $\left|\phi_{-}\right\rangle$eigenstates. The numerical results in Fig. 2(b) show an eigenstate energy difference of $37.6 \mathrm{meV}$, which corresponds to a time scale of $110 \mathrm{fs}$. This time scale matches very well with the ion yield oscillation observed in the experimental results of Fig. 2(a).

We can now model the observed features in the $\mathrm{CO}^{+}$ion yield by accounting for the NIR probing step and the rotational degrees of freedom in the molecular system. Assuming a dominant $s$-wave nature for photoionization, dipole selection rules dictate that the ionization will preferentially occur from $3 \sigma_{u}$ orbitals for molecules aligned parallel to the XUV field [29]. Therefore, the XUV pulse prepares an aligned distribution of $\mathrm{CO}_{2}^{+}$ions in a $B^{2} \Sigma_{u}^{+}$ electronic configuration. Further, due to the dipole selection rules and bandwidth constraints, the parallel NIR probing field exclusively maps the $B^{2} \Sigma_{u}^{+}$state population to the predissociating $C^{2} \Sigma_{g}^{+}$state [38], resulting in the formation of $\mathrm{CO}^{+}$ions. This transition is forbidden for a perpendicular probing field, explaining the large discrepancy in ion yield between the parallel and perpendicular polarizations at $t=0 \mathrm{fs}$ seen in Fig. 2(a).

To incorporate ionization induced alignment and thermal rotations into our simulation, we perform a quantumclassical trajectory calculation [29]. We compute the ensemble-averaged NIR transition dipole $D_{B}(t)$ from the $B^{2} \Sigma_{u}^{+}$state to the final $C^{2} \Sigma_{g}^{+}$state. Using the timedependent wave function in Eq. (2) and calculating $D_{B}(t)$, we obtain the time-dependent $\mathrm{CO}^{+}$ion yield as [29]

$$
\begin{aligned}
I\left(\mathrm{CO}^{+}, t\right) \propto & \left(\cos ^{4} \theta+\sin ^{4} \theta\right) D_{B}(t)^{2} \\
& +\frac{1}{2} \sin ^{2} 2 \theta D_{B}(t)^{2} \cos \left(E_{+}-E_{-}\right) t .
\end{aligned}
$$

The 110 fs quantum beat is, therefore, reproduced in the experimental $\mathrm{CO}^{+}$ion yield. Additionally, the finite rotational temperature $T_{\text {rot }}$ of the molecular ensemble leads to the loss of the cation's alignment, resulting in a time dependent $D_{B}(t)$. Figure 2(c) shows the result of Eq. (3) for a rotational temperature relevant to our experimental conditions $\left(T_{\text {rot }}=200 \mathrm{~K}\right)$. The computed signal intensity for parallel NIR polarization undergoes a decay in nearly 400 fs, which matches the experimental observation in Fig. 2(a). This decay can be explained as follows: as the ensemble rotates out of alignment, a smaller fraction of the molecules can be probed by the parallel NIR field. On the other hand, this opens up a larger number of molecules which can be probed by the perpendicular NIR field, resulting in the increase in ion yield for perpendicular polarization.

With strong agreement between experiment and theory, we finally arrive at a complete picture of coupled nuclearhole dynamics near a conical intersection (Fig. 3). The XUV pulse first prepares the molecule in a coherent superposition of cationic eigenstates with an initial $\sigma_{u}$ electron hole character. The bending and asymmetric stretch motions of the molecule coherently drive the electron hole density from the region along the molecular axis with $\sigma_{u}$ symmetry, to the region around the molecular axis with $\pi_{u}$ symmetry. The relative electron hole density corresponding to the evolution of the wave packet within the first $110 \mathrm{fs}$ period of quantum beating is shown in Fig. 3(a). The reduced density of the nuclear wave packet

(a)

$\Delta \rho_{\text {hole }}$
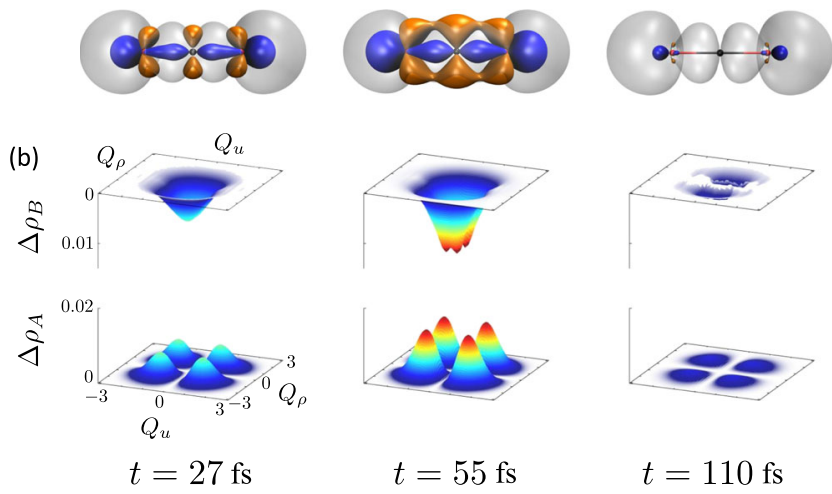

FIG. 3 (color online). (a) Change in electron hole density $\Delta \rho_{\text {hole }}$ with respect to time zero. The dynamics shown correspond to the $110 \mathrm{fs}$ quantum beat. During this period, the electron hole density is driven from $\sigma_{u}$ character along the molecular axis to $\pi_{u}$ character around the molecular axis. The blue (darker shading) represents a negative change and the orange (lighter shading) represents a positive change. (b) Change in the reduced density of the vibronic wave packet along the coupling coordinates $Q_{u}$ and $Q_{\rho}$ associated with electronic states $B^{2} \Sigma_{u}^{+}$(upper panel) and $A^{2} \Pi_{u}$ (lower panel). The nuclear dynamics corresponding to the bending and asymmetric stretching motion occur in sync with the electron hole motion. 
(a)

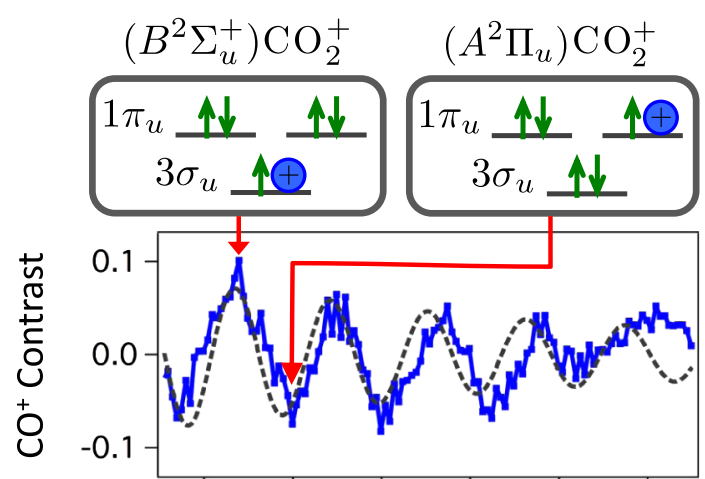

(b)

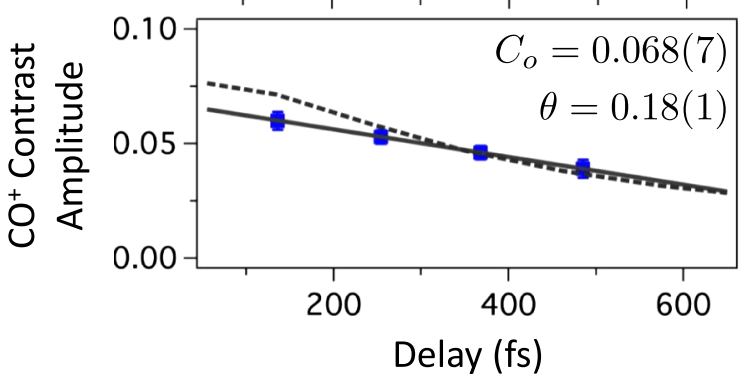

FIG. 4 (color online). (a) The experimental results for the contrast of $\mathrm{CO}^{+}$ion yield oscillation for parallel polarization. The contrast peaks and minima reflect the electronic character of the hole as it evolves in time. The dashed curve is the theoretically calculated contrast incorporating the effects of dephasing due to slightly different rotational constants of the $A^{2} \Pi_{u}$ and $B^{2} \Sigma_{u}^{+}$states and the rotational temperature of the ensemble. (b) The contrast amplitude is plotted for the four prominent oscillation periods shown in (a) and it decays linearly over time due to the loss of coherence. A linear fit to this data allows us to experimentally measure the mixing angle to be $\theta=0.18(1)$ before the onset of decoherence. The dashed line is the theoretical calculated decay of contrast amplitude.

on the $A^{2} \Pi_{u}$ and $B^{2} \Sigma_{u}^{+}$states in terms of the asymmetric stretching $\left(Q_{u}\right)$ and bending $\left(Q_{\rho}\right)$ coordinates is shown in Fig. 3(b) and demonstrates periodic modulation in synchronization with the electron hole dynamics.

We can also monitor the evolution of quantum coherence in the cationic superposition by using the results in Fig. 2(a) and defining the time-dependent ion yield contrast as

$$
C_{\text {expt }}(\tau)=\frac{I_{\mathrm{sig}}(\tau)-I_{\mathrm{dc}}(\tau)}{I_{\mathrm{dc}}(\tau)},
$$

where $I_{\text {sig }}(\tau)$ is the raw $\mathrm{CO}^{+}$ion yield and $I_{\mathrm{dc}}(\tau)$ is the nonoscillatory component. In Fig. 4(a), we plot the delaydependent contrast observed in the experiment. We can compare this to the theoretical ion-yield contrast from Eq. (3), or

$$
C_{\text {theo }}(t)=\frac{\frac{1}{2} \sin ^{2}(2 \theta)}{\cos ^{4} \theta+\sin ^{4} \theta} \cos \left(E_{+}-E_{-}\right) t .
$$

The amplitude of the theoretical contrast depends only on the mixing angle and is stationary with time, whereas the amplitude of the experimental contrast in Fig. 4(a) decays as a function of time delay. This decay in amplitude is indicative of the loss of coherence. In Fig. 4(b), we plot the delay-dependent contrast amplitude for the four prominent oscillation periods observed in the experiment and find that the contrast amplitude decreases linearly with time. Using linear extrapolation, we obtain the initial contrast amplitude before the onset of decoherence, $C_{o}$, corresponding to the oscillation peak at $t=0 \mathrm{fs}$. Equating $C_{o}$ to the analytical expression for the contrast amplitude [Eq. (5)], we obtain an experimental measurement of the mixing angle, $\theta=0.18(1)$. This can readily be compared with the mixing angle directly obtained from the numerical results of the MCTDH calculations which yield $\theta=0.195$ [29]. Our paper, thus, represents a sensitive measurement of the coupling induced by the nonadiabatic perturbations near a conical intersection.

From the linear fit in Fig. 4(b), we experimentally infer the rate of decoherence to be $(0.06 \pm 0.01) \mathrm{ps}^{-1}$. The mechanism behind this can be understood in terms of thermal dephasing. At a finite rotational temperature, XUV excitation from the ground state creates an incoherent distribution of rotational levels in the $\left|\chi_{B}\right\rangle$ vibronic state. Since the rotational constants of coupled $\left|\chi_{B}\right\rangle$ and $\left|\chi_{A}\right\rangle$ states are appreciably different, the energy gap between them varies with the rotational quantum number, $J$ [29]. As a result, the $J$-dependent phases of electron hole oscillations diverge with time, manifesting as a loss of coherence in our data. The dashed curve in Fig. 4(a) represents the results of a semiclassical, linear rotor model incorporating the effects of rotational dephasing of a thermal distribution with $T_{\text {rot }}=200 \mathrm{~K}$. The numerical contrast decays on a very similar time scale to what is seen in the experiment. This is further exemplified in Fig. 4(b), where the theoretical contrast amplitude (dashed line) follows the experimental amplitude (solid line). It is interesting to note that while the thermal ensemble is fairly warm, the coherence in the weakly coupled cationic states persists for more than $500 \mathrm{fs}$. The concept of quantum coherence is playing an increasingly important role in our understanding of chemical and biological phenomena [39,40], and our results suggest that the nonadiabatic coupling to nuclear motion provides an efficient means for mediating coherent charge transfer in polyatomic systems.

In conclusion, we used an XUV pump and NIR probe to resolve the ultrafast motion of an electron hole between $\sigma$ and $\pi$ orbitals in the $\mathrm{CO}_{2}$ molecule. We quantified the coupling between electronic states due to the perturbation caused by the conical intersection and measured the evolution of quantum coherence during the charge oscillation. This approach is quite general and can be easily extended to resolve the elusive electronic correlation driven charge migration dynamics [41]. The real-time visualization of electron hole dynamics in such nonadiabatic scenarios and the understanding of the limitations of the 
quantum system due to coupling with environmental degrees of freedom is fundamental in probing the inner workings of charge transfer processes occurring in nature. The experimental and theoretical results we obtained here for the linear triatomic molecule represent first steps in elucidating these dynamics and pave the way for the application of attosecond and femtosecond XUV spectroscopy in the measurement and control of charge dynamics in complex biochemical processes.

H. T., N. S., and A. S. acknowledge the support from the National Science Foundation (Grant No. PHY-0955274) and an Arizona TRIF Photonics Fellowship.

*sandhu@physics.arizona.edu

[1] V. May and O. Kühn, Charge and Energy Transfer Dynamics in Molecular Systems, 3rd ed. (Wiley-VCH, Weinheim, 2011).

[2] V. Sundstrom, Annu. Rev. Phys. Chem. 59, 53 (2008).

[3] H. Wang, M. Chini, S. Chen, C.-H. Zhang, Y. Cheng, F. He, Y. Wu, U. Thumm, and Z. Chang, Phys. Rev. Lett. 105, 143002 (2010).

[4] C. Ott, A. Kaldun, P. Raith, K. Meyer, M. Laux, J. Evers, C. H. Keitel, C. H. Greene, and T. Pfeifer, Science 340, 716 (2013).

[5] E. Goulielmakis, Z. Loh, A. Wirth, R. Santra, N. Rohringer, V. Yakovlev, S. Zherebtsov, T. Pfeifer, A. Azzeer, M. Kling, S. Leone, and F. Krausz, Nature (London) 466, 739 (2010).

[6] M. Schultze et al., Science 328, 1658 (2010).

[7] E. Gagnon, P. Ranitovic, X. M. Tong, C. L. Cocke, M. M. Murnane, H. C. Kapteyn, and A. S. Sandhu, Science 317, 1374 (2007).

[8] M. F. Lin, D. M. Neumark, O. Gessner, and S. R. Leone, J. Chem. Phys. 140, 064311 (2014).

[9] H. Timmers, N. Shivaram, and A. Sandhu, Phys. Rev. Lett. 109, 173001 (2012).

[10] H. Wörner, J. Bertrand, B. Fabre, J. Higuet, H. Ruf, A. Dubrouil, S. Patchkovskii, M. Spanner, Y. Mairesse, V. Blanchet, E. Mével, E. Constant, P. Corkum, and D. Villeneuve, Science 334, 208 (2011).

[11] K. P. Singh, F. He, P. Ranitovic, W. Cao, S. De, D. Ray, S. Chen, U. Thumm, A. Becker, M. M. Murnane, H. C. Kapteyn, I. V. Litvinyuk, and C. L. Cocke, Phys. Rev. Lett. 104, 023001 (2010).

[12] A. Fischer, A. Sperl, P. Corlin, M. Schonwald, H. Rietz, A. Palacios, A. Gonzalez-Castrillo, F. Martin, T. Pfeifer, J. Ullrich, A. Senftleben, and R. Moshammer, Phys. Rev. Lett. 110, 213002 (2013).

[13] B. Erk et al., Phys. Rev. Lett. 110, 053003 (2013).

[14] B. Cooper and V. Averbukh, Phys. Rev. Lett. 111, 083004 (2013).

[15] A. I. Kuleff, J. Breidbach, and L. S. Cederbaum, J. Chem. Phys. 123, 044111 (2005).

[16] L. Lehr, T. Horneff, R. Weinkauf, and E. Schlag, J. Phys. Chem. A 109, 8074 (2005).
[17] S. Pabst, L. Greenman, P. J. Ho, D. A. Mazziotti, and R. Santra, Phys. Rev. Lett. 106, 053003 (2011).

[18] M. S. Schoffler et al., Science 320, 920 (2008).

[19] T. Jahnke, H. Sann, T. Havermeier, K. Kreidi, C. Stuck, M. Meckel, M. Schöffler, N. Neumann, R. Wallauer, S. Voss, A. Czasch, O. Jagutzki, A. Malakzadeh, F. Afaneh, T. Weber, H. Schmidt-Böcking, and R. Dörner, Nat. Phys. 6, 139 (2010).

[20] G. Worth and L. Cederbaum, Annu. Rev. Phys. Chem. 55, 127 (2004).

[21] L. Wang, J. Reutt, Y. Lee, and D. Shirley, J. Electron Spectrosc. Relat. Phenom. 47, 167 (1988).

[22] T. Zimmermann, H. Köppel, and L. Cederbaum, J. Chem. Phys. 83, 4697 (1985).

[23] J. Liu, M. Hochlaf, and C. Ng, J. Chem. Phys. 113, 7988 (2000).

[24] T. S. Rose, M. J. Rosker, and A. H. Zewail, J. Chem. Phys. 88, 6672 (1988).

[25] P. Farmanara, V. Stert, and W. Radloff, J. Chem. Phys. 111, 5338 (1999).

[26] C. Z. Bisgaard, O. J. Clarkin, G. Wu, A. Lee, O. Gessner, C. C. Hayden, and A. Stolow, Science 323, 1464 (2009).

[27] A. E. Boguslavskiy, M. S. Schuurman, D. Townsend, and A. Stolow, Faraday Discuss. 150, 419 (2011).

[28] M. Beck, A. Jäckle, G. Worth, and H. Meyer, Phys. Rep. 324, 1 (2000).

[29] See Supplemental Material at http://link.aps.org/ supplemental/10.1103/PhysRevLett.113.113003 for details of the experimental set up and theoretical calculation procedures, which includes Refs. [30-37].

[30] F. J. Furch, S. Birkner, J. H. Jungmann, F. Kelkensberg, C. P. Schulz, A. Rouzee, and M. J. J. Vrakking, J. Chem. Phys. 139, 124309 (2013).

[31] Y. Lin, P. Pieniazek, M. Yang, and J. Skinner, J. Chem. Phys. 132, 174505 (2010).

[32] E. Smith and D. Jones, J. Phys. Chem. A 115, 4101 (2011).

[33] J. Tully, J. Chem. Phys. 93, 1061 (1990).

[34] S. Hammes-Schiffer and J. Tully, J. Chem. Phys. 101, 4657 (1994).

[35] D. A. Shaw, D. M. P. Holland, M. A. Hayes, M. A. MacDonald, A. Hopkirk, and S. M. McSweeney, Chem. Phys. 198, 381 (1995).

[36] M. W. Schmidt, K. K. Baldridge, J. A. Boatz, S. T. Elbert, M. S. Gordon, J. H. Jensen, S. Koseki, N. Matsunaga, K. A. Nguyen, S. J. Su, T. L. Windus, M. Dupuis, and J. A. Montgomery, J. Comput. Chem. 14, 1347 (1993).

[37] C. M. Oana and A. I. Krylov, J. Chem. Phys. 127, 234106 (2007).

[38] J. Liu, W. Chen, M. Hochlaf, X. Qian, C. Chang, and C. Ng, J. Chem. Phys. 118, 149 (2003).

[39] N. Lambert, Y. nan Chen, Y.-C. Cheng, C.-M. Li, G.-Y. Chen, and F. Nori, Nat. Phys. 9, 10 (2013).

[40] G. S. Engel, T. R. Calhoun, E. L. Read, T. Ahn, T. Mancal, Y. Cheng, R. Blankenship, and G. Fleming, Nature (London) 446, 782 (2007).

[41] J. Breidbach and L. Cederbaum, J. Chem. Phys. 118, 3983 (2003). 